\title{
Improved Delineation of Arteries in the Posterior Fossa of the Brain by Model-Based Iterative Reconstruction in Volume-Rendered 3D CT Angiography
}

\author{
H. Machida, H. Takeuchi, I. Tanaka, R. Fukui, Y. Shen, E. Ueno, S. Suzuki, and X.-Z. Lin
}

\begin{abstract}
BACKGROUND AND PURPOSE: Improved CTA delineation of arteries and unruptured aneurysms is clinically desired in the posterior fossa. We present a novel model-based iterative reconstruction that models system statistics and optics to improve CT image quality. We investigated the utility of MBIR for improving delineation of arteries in the posterior fossa on 3D brain CTA.
\end{abstract}

MATERIALS AND METHODS: Using filtered back-projection with a standard kernel and MBIR, we reconstructed axial images of 0.625-mm thickness of 28 consecutive patients (14 men; mean age, $58.6 \pm 14.6$ years) who underwent 64-detector brain CTA. We placed regions of interest on the axial images, measured the mean CT value in the basilar artery and the value and SD in the pons and bilateral cerebellar hemispheres, and calculated the contrast-to-noise ratio of the brain arteries in the posterior fossa. Using volume-rendered CTA and a 4-point scale, 2 radiologists independently graded delineation of the BA, bilateral vertebral artery, superior cerebellar artery, and anterior and posterior inferior cerebellar arteries. We compared the results between FBP and MBIR by using paired $t$ and Wilcoxon signed-rank tests.

RESULTS: Compared with FBP, MBIR significantly improved the contrast-to-noise ratio $(P<.0001)$ and subjective delineation of all arteries in the posterior fossa except the BA (VA, SCA, AICA, and PICA; $P<.05$ for all). The mean visual score by MBIR was 3.0 or higher for all those arteries except the AICA assessed by reader $1(2.6 \pm 0.7)$.

CONCLUSIONS: With 3D brain CTA, contrast-to-noise ratio and arterial delineation of the VA, SCA, AICA, and PICA in the posterior fossa are better with MBIR than FBP.

ABBREVIATIONS: AICA = anterior inferior cerebellar artery; $\mathrm{BA}=$ basilar artery; $\mathrm{FBP}=$ filtered back-projection; $\mathrm{MBIR}=$ model-based iterative reconstruction; SCA = superior cerebellar artery; $\mathrm{VA}=$ vertebral artery

$\mathrm{T}$ he standard filtered back-projection algorithm with CT assumes perfect projection samples by ignoring the noise inherent in x-ray attenuation and detector electronics and assumes ideal system optics by featuring point sources, voxels, and detector elements. Model-based iterative reconstruction, a clinically available state-of-the-art comprehensive algorithm for CT, models system noise statistics and 3D system optics of the specific CT scanner hardware for which it was developed. Phantom studies have confirmed enhanced image resolution, lower noise, and re-

Received February 13, 2012; accepted after revision July 29.

From the Department of Radiology (H.M., I.T., R.F., Y.S., E.U.), Tokyo Women's Medical University Medical Center East, Tokyo, Japan; Department of Radiology (H.T.), Tokyo Metropolitan Tama Medical Center, Tokyo, Japan; Department of Radiology (S.S.), Saitama Red Cross Hospital, Saitama, Japan; and Department of Radiology (X.-Z.L.), Shanghai Jiaotong University School of Medicine, Shanghai, China.

Please address correspondence to Haruhiko Machida, MD, Tokyo Women's Medical University Medical Center East, 2-1-10 Nishiogu, Arakawa-ku, Tokyo 116-8567,

Japan; e-mail: machira@dnh.twmu.ac.jp

http://dx.doi.org/10.3174/ajnr.A3320 duced helical conebeam artifacts by using the MBIR algorithm. ${ }^{1}$ Thus, its use is expected to improve image quality and enable reduced doses of radiation and contrast medium in CT studies in various clinical fields.

3D CT angiography can be used to guide the treatment strategy by permitting precise assessment of the size and shape of intracranial aneurysms and their anatomic relationships with parent vessels. ${ }^{2-6}$ Especially, the use of the volume-rendered technique maintains the original anatomic spatial relationships of the CT dataset and offers a 3D perspective to facilitate interpretation of vascular interrelationships. At least $10 \%$ of unruptured intracranial aneurysms occur in the vertebrobasilar system, a location reported to be a significant independent predictor of aneurysm rupture. ${ }^{7,8}$ On the other hand, posterior fossa artifacts and enhanced image noise can hinder delineation of those aneurysms. Thus, we compared delineation of the arteries in the posterior fossa and vertebrobasilar system on volumerendered 3D CTA between MBIR and FBP to assess whether MBIR achieved more useful results. 


\section{MATERIALS AND METHODS Study Population}

From March 10 through April 7, 2011, we retrospectively evaluated images of 28 consecutive patients ( 14 men, 14 women; 26-80 years of age; mean age, $58.6 \pm 14.6$ years) who underwent CTA of the brain arteries by using a garnet-based 64-detector high-definition CT scanner (Discovery CT750 HD; GE Healthcare, Milwaukee, Wisconsin) and our standard protocol. The CTA examinations were performed for further evaluation of unruptured intracranial aneurysms (14 patients), intracerebral hemorrhage $(n=4)$, subarachnoid hemorrhage $(n=3)$, cerebral infarction $(n=3)$, and Moyamoya disease $(n=1)$ and after coil embolization for saccular aneurysms of the internal carotid artery $(n=3)$. Tokyo Women's Medical University institutional review board waived informed patient consent for this retrospective study, but all patients provided written informed consent for the CTA examinations.

\section{Image Data Acquisition}

To minimize motion artifacts, patients underwent CTA examination with their heads immobilized by using a head holder. For the contrast-enhanced helical CT scan, parameters were the following: tube voltage, $120 \mathrm{kV}$; tube current, $335 \mathrm{~mA}$; rotation speed, 0.5 seconds; and pitch, 0.516 . Patients received a bolus of contrast medium with iodine concentration of $370 \mathrm{mgI} / \mathrm{mL}(50 \mathrm{~mL}$ of iopamidol; Iopamiron, Bayer HealthCare, Osaka, Japan) via a 20-ga intravenous catheter placed in an antecubital vein at a rate of $4.0 \mathrm{~mL} / \mathrm{s}$ followed by a $30-\mathrm{mL}$ saline bolus at the same rate by using a double-head power injector (Dual Shot, type GX; Nemoto Kyorindo, Tokyo, Japan). To determine the scanning delay, a test bolus of $10 \mathrm{~mL}$ of contrast medium and $15 \mathrm{~mL}$ of saline was injected at the same rate. For each patient, a circular region of interest was placed within the A1/A2 portion of the anterior cerebral artery to determine the peak time of attenuation, and the CT scan was started 2 seconds after that peak. The patient underwent scanning in the craniocaudal direction from the top of the cranial vault to the bottom of the anterior arch of the first cervical vertebra. Helical data were reconstructed in the axial plane (FOV, $20 \mathrm{~cm}$ ) with a section thickness of $0.625 \mathrm{~mm}$ at $0.625-\mathrm{mm}$ intervals using FBP with a standard kernel (matrix size, $512 \times 512$ ) and MBIR (matrix size, $1024 \times 1024)$ and then were transferred to a workstation (Advantage Windows 4.5; GE Healthcare). This MBIR algorithm (Veo; GE Healthcare) was clinically available for cranial CT or CTA examinations only with a matrix size of $1024 \times 1024$.

On the workstation, 2 trained radiology technologists generated volume-rendered 3D CTA images by using a standard technique. The same parameters for volume-rendered reconstruction were used for all patients and both reconstruction algorithms: a linear threshold with a lower threshold value of $125 \mathrm{HU}$ set at $0 \%$ opacity (completely transparent) and an upper threshold of $600 \mathrm{HU}$ set at $100 \%$ opacity (completely opaque). Bone structures were not edited out of the axial images. The 3D CTA images of the posterior fossa of each patient were extracted by using an $8-\mathrm{cm}$ FOV and were displayed in a view from behind with superoinferior angulation of approximately $30^{\circ}$; for comparison, identical images reconstructed by FBP and MBIR were arranged side by side on the workstation.

\section{Quantitative Assessment}

For each patient, the 2 radiology technologists placed circular ROIs of uniform size within the pons, bilateral cerebellar hemispheres, and basilar artery on the reconstructed axial images of 0.625 -mm thickness to measure the attenuation value and its SD with the 2 different reconstruction algorithms. For each reconstruction method, we calculated the mean attenuation value of the BA; used the averaged attenuation value in the pons and bilateral cerebellar hemispheres as the attenuation value in posterior fossa, and the averaged SD value in the pons and bilateral cerebellar hemispheres as the objective noise in the posterior fossa and calculated the contrast-to-noise ratio as contrast-to-noise ratio $=($ attenuation value in $\mathrm{BA}-$ attenuation value in posterior fossa) / objective noise in posterior fossa.

We recorded the volume CT dose index (measured in milligrays) and dose-length product (measured in milligrays $\times$ centimeter) displayed on the dose report on the CT scanner for each patient and calculated the mean volume CT dose index and doselength product in our patients.

\section{Qualitative Assessment}

Two board-certified radiologists experienced in interpreting CTA of the brain with 8 and 13 years' cumulative experience (H.T. [reader 1] and H.M. [reader 2]), respectively, and blinded to information about patients and reconstruction methods independently performed visual evaluation of not only volume-rendered but also coronal maximum-intensity-projection CTA images of each patient in the posterior view obtained by using the 2 different reconstruction algorithms and displayed side by side at the workstation. For the maximumintensity-projection reconstruction, they used the same parameters for all patients and both reconstruction algorithms (slab thickness, 10 $\mathrm{mm}$; FOV, $8 \mathrm{~cm}$; window level, $300 \mathrm{HU}$; and window width, $900 \mathrm{HU}$ ) and viewed these images while scrolling. They focused on delineation of the arteries in the posterior fossa: the BA and bilateral vertebral artery and their branches, the bilateral superior cerebellar artery, and the anterior and posterior inferior cerebellar arteries. Visual evaluation was generally performed at a fixed view, with the viewing direction adjusted only to avoid superimposition of vessels and to confirm anatomy, if necessary. They scored the images on a 4-point scale (4, excellent; 3 , good; 2 , fair; 1 , poor) with regard to vessel depiction and contrast, superimposed image noise, and sharpness of vessel contour. Scores of 2, 3, and 4 represented acceptable image quality, and a score of 1 point, unacceptable quality.

\section{Statistical Analysis}

All continuous variables were expressed as mean \pm SD. We performed statistical analysis by using StatView for Windows (Version 5.0; SAS Institute, Cary, North Carolina). We used a paired $t$ test to compare objective results and the Wilcoxon signed-rank test to compare subjective results between the FBP and MBIR reconstruction algorithms. We assessed inter-reader agreement of the visual evaluation scores by using Cohen $\kappa$ statistics. $P<.05$ was considered statistically significant.

\section{RESULTS}

\section{Quantitative Results}

The mean volume CT dose index was $56.2 \pm 0.1 \mathrm{mGy}$, and the mean dose-length product, $983.7 \pm 50.2 \mathrm{mGy} \times \mathrm{cm}$, based on the imaging parameters for 3D CT angiography of the brain arteries. 
Table 1: Quantitative assessment ${ }^{\mathrm{a}}$

\begin{tabular}{lccc}
\hline & FBP & MBIR & $P$ Value \\
\hline BA attenuation $(\mathrm{HU})$ & $422.0 \pm 93.3$ & $466.1 \pm 90.5$ & $<.0001$ \\
Image noise $(\mathrm{HU})$ & $10.1 \pm 1.3$ & $8.8 \pm 0.7$ & $<.0001$ \\
CNR & $37.9 \pm 11.0$ & $48.2 \pm 10.1$ & $<.0001$ \\
\hline
\end{tabular}

Note:-CNR indicates contrast-to-noise ratio.

${ }^{a}$ Data are means.

Table 1 summarizes the results of quantitative assessment. MBIR significantly improved the attenuation value of the BA, image noise, and contrast-to-noise ratio $(466.1 \pm 90.5 \mathrm{HU}, 8.8 \pm$ $0.7 \mathrm{HU}$, and $48.2 \pm 10.1)$ compared with FBP $(422.0 \pm 93.3 \mathrm{HU}$, $10.1 \pm 1.3 \mathrm{HU}$, and $37.9 \pm 11.0)(P<.0001$ for all $)$.

\section{Qualitative Results}

Table 2 summarizes the results of the qualitative assessment.

On volume-rendered images, the mean visual evaluation score by both readers decreased in order from the BA and VA to the PICA to the SCA to the AICA by using both FBP and MBIR algorithms. The scores for the BA and VA by MBIR were 3 or 4 for both readers in all patients and by FBP in 27 of 28 patients (96\%). Compared with FBP, MBIR significantly improved subjective delineation of all brain arteries in the posterior fossa except the BA (SCA, AICA, and PICA: $P<.0001$ for both the readers; VA: $P=$ .046 [reader 1] and $P=.008$ [reader 2]). The mean visual score by MBIR was 3.0 or higher for all those arteries except the AICA as assessed by reader $1(2.6 \pm 0.7)$. Image quality was acceptable (score 2, 3, or 4) for most arteries in the posterior fossa by MBIR; Reader 1 scored only 1 SCA image as unacceptable. The interreader agreement of the visual scores was moderate ( $\kappa$ value, 0.43 [95\% confidence interval, $0.36-0.50]$ ).

On maximum intensity projections, the mean visual evaluation score by both readers decreased in order from the BA and VA to the SCA and PICA to the AICA by using both FBP and MBIR algorithms. Compared with FBP, MBIR significantly improved subjective delineation of all brain arteries, particularly larger arteries such as the BA and VA, in the posterior fossa $(P<.0001$ for both the readers). The averaged improvement of the visual score by MBIR compared with FBP was 0.84 for the BA and VA and 0.46 for the SCA, AICA, and PICA. The mean visual score by MBIR was 3.0 or higher for all those arteries except the AICA $(2.8 \pm 0.7$ by both the readers), whereas the score by FBP was 3.0 or higher only for the BA and VA. Image quality was acceptable for all the arteries in the posterior fossa by both FBP and MBIR. The interreader agreement of the visual scores was substantial ( $\kappa$ value, 0.75 [95\% confidence interval, $0.69-0.81]$ ).

In images of the posterior fossa in a 69-year-old woman with a basilar apex aneurysm, volume-rendered 3D CTA by using the 2 different reconstruction algorithms demonstrated particularly improved delineation of the bilateral AICA and SCA by MBIR compared with FBP, with comparable delineation of the BA and bilateral VA between the 2 algorithms (Fig 1). On maximum intensity projections, MBIR improved subjective delineation of all brain arteries in the posterior fossa, including the BA, with improved vessel enhancement and sharpness of vessel contour, compared with FBP (Fig 1).

\section{DISCUSSION}

At least $10 \%$ of unruptured intracranial aneurysms occur in the vertebrobasilar system, a location reported as a significant independent predictor of aneurysm rupture. ${ }^{7,8}$ Many aneurysms in this location are detected only after $\geq 2$ digital subtraction angiography studies. ${ }^{9-11}$ BA aneurysms masked by crossflow phenomena and peripherally located aneurysms of the posterior fossa with reduced filling flow can frequently be overlooked at the first DSA study. Peripherally located aneurysms of the posterior fossa are not rare, ${ }^{12}$ but some can be detected only with brain CTA. ${ }^{13,14}$ Volume-rendered 3D CTA can provide complete quantification and characterization of intracranial aneurysms and important information for treatment planning. ${ }^{2-6}$

Increased image noise and artifacts can degrade CTA delineation of brain arteries, especially small vessels, in the posterior fossa. ${ }^{15,16}$ Use of higher spatial resolution to improve small-vessel depiction leads to increased noise; use of higher tube current to reduce noise increases radiation exposure; use of a high dose of contrast to improve arterial enhancement and thus the contrastto-noise ratio may increase the risk of contrast-induced nephropathy in patients with marginal renal function; and a rapid injection rate increases the risk of extravasation at the injection site. In large part, posterior fossa artifacts represent beam-hardening artifacts and nonlinear partial volume effects caused by the thick irregular bone of the skull base. ${ }^{17-19}$ Previous studies have used a variety of technologies to decrease these artifacts, including the use of newer multidetector CT machines using a helical scan technique with thinner image collimation, higher output tubes, and better reconstruction algorithms (such as beam-hardening correction and iterative reconstruction). ${ }^{16-18,20-23}$ The polychromaticity of $\mathrm{x}$-rays causes beam-hardening artifacts in images and loss of information from energy averaging, but monochromatic images produced with CT spectral imaging have recently been reported to reduce beam-hardening artifacts and image noise in the posterior fossa. ${ }^{24}$ On the other hand, iterative reconstruction can recover the attenuation map ${ }^{23}$ and was confirmed to be robust and effective for beam-hardening correction. ${ }^{25}$

MBIR is a comprehensive state-of-the-art iterative algorithm for image reconstruction for clinical CT study that has the potential to improve image quality with respect to noise, spatial resolution, and helical conebeam artifacts ${ }^{1}$ and to reduce radiation and the contrast-medium dose, with only the sacrifice of a long computing time in the present state. Specifically, the average reconstruction time for CTA of the brain with MBIR was approximately 1 hour in this study. Different from FBP, MBIR models the statistical behavior of measurements (photon statistics and electronic noise) and detector response to incident photons (system optics). The latter model describes how each element of the object illuminated by $\mathrm{x}$-ray from the source is projected onto the detector. Each voxel element of the scanned object is represented by a finite $3 \mathrm{D}$ volume, and voxels are illuminated by a finite beam that stems from a focal spot of known dimensions and that is measured over the active area of the detector. This spatially varying behavior better models the real CT system than the point model of FBP and leads to better information recovery in the CT image.

In phantom experiments, MBIR achieved only slightly lower through-plane resolution than FBP with half the detector aper- 
Table 2: Qualitative assessment ${ }^{\mathrm{a}}$

\begin{tabular}{lccccccc}
\hline & \multicolumn{3}{c}{ VR } & & & \multicolumn{3}{c}{ MIP } \\
\cline { 2 - 3 } & FBP & MBIR & P Value & & FBP & MBIR & P Value \\
\hline BA (reader 1) & $3.9 \pm 0.4$ & $4.0 \pm 0.2$ & .083 & & $3.0 \pm 0.3$ & $3.8 \pm 0.4$ & $<.0001$ \\
BA (reader 2) & $3.9 \pm 0.3$ & $4.0 \pm 0.0$ & .083 & & $3.0 \pm 0.2$ & $3.9 \pm 0.3$ & $<.0001$ \\
VA (reader 1) & $3.9 \pm 0.3$ & $4.0 \pm 0.1$ & .046 & & $3.0 \pm 0.2$ & $3.7 \pm 0.5$ & $<.0001$ \\
VA (reader 2) & $3.9 \pm 0.3$ & $4.0 \pm 0.0$ & .008 & & $3.0 \pm 0.0$ & $3.9 \pm 0.4$ & $<.0001$ \\
SCA (reader 1) & $2.1 \pm 0.7$ & $3.0 \pm 0.6$ & $<.0001$ & & $2.8 \pm 0.5$ & $3.1 \pm 0.6$ & $<.0001$ \\
SCA (reader 2) & $2.5 \pm 0.9$ & $3.6 \pm 0.7$ & $<.0001$ & & $2.8 \pm 0.4$ & $3.4 \pm 0.7$ & $<.0001$ \\
AICA (reader 1) & $1.6 \pm 0.7$ & $2.6 \pm 0.7$ & $<.0001$ & & $2.4 \pm 0.5$ & $2.8 \pm 0.7$ & $<.0001$ \\
AICA (reader 2) & $1.7 \pm 0.9$ & $3.2 \pm 0.9$ & $<.0001$ & & $2.4 \pm 0.5$ & $2.8 \pm 0.7$ & $<.0001$ \\
PICA (reader 1) & $2.7 \pm 0.6$ & $3.3 \pm 0.5$ & $<.0001$ & & $2.7 \pm 0.5$ & $3.3 \pm 0.6$ & $<.0001$ \\
PICA (reader 2) & $2.9 \pm 0.8$ & $3.8 \pm 0.4$ & $<.0001$ & & $2.8 \pm 0.4$ & $3.3 \pm 0.7$ & $<.0001$ \\
\hline
\end{tabular}

Note:-VR indicates volume-rendered; MIP, maximum intensity projection.

${ }^{a}$ Data are means.
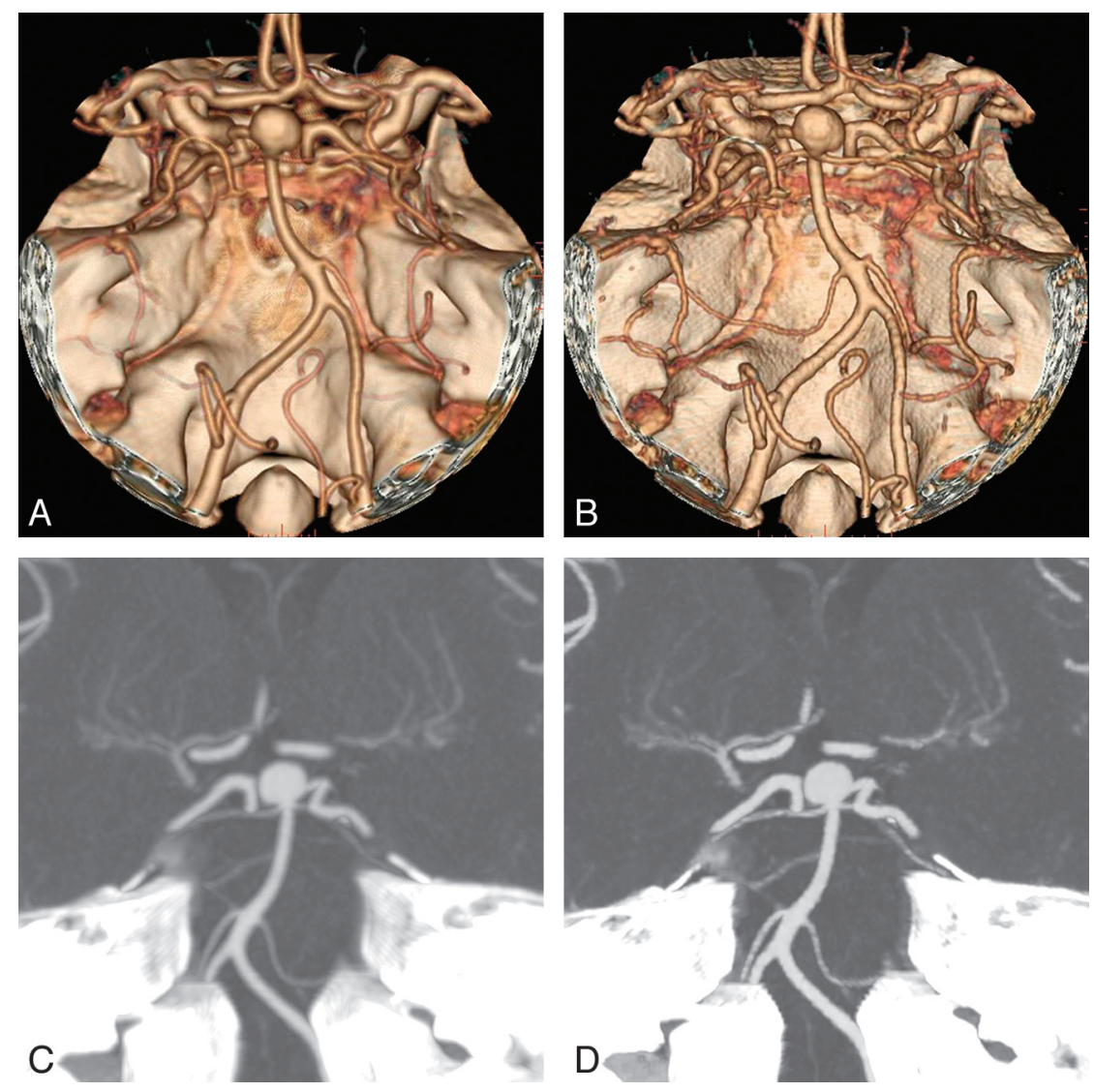

FIG 1. A 69-year-old woman with a basilar apex aneurysm. Volume-rendered (VR) 3D CTAs of the posterior fossa by using 2 different reconstruction algorithms, filtered back-projection (FBP) with a "standard" kernel $(A)$ and model-based iterative reconstruction (MBIR) $(B)$, and oblique coronal maximum-intensity-projection (MIP) CTAs by using FBP with a "standard" kernel (C) and MBIR (D). On VR, delineation of the bilateral anterior inferior cerebellar (AICA) and superior cerebellar (SCA) arteries is particularly improved by MBIR compared with FBP (reader 1: right AICA, 3 versus 3; left AICA, 3 versus 2; and bilateral SCA, 2 versus 2; reader 2: right AICA, 4 versus 3; left AICA, 4 versus 1; right SCA, 4 versus 2; and left SCA, 3 versus 2); and delineation of the basilar (BA) and bilateral vertebral arteries (VA) is comparable between the 2 algorithms (readers 1 and 2: the BA and bilateral VA, 4 versus 4). On MIP, delineation of the BA (readers 1 and 2 : four versus 3 ) and the AICA (reader 1: right AICA, 4 versus 3; left AICA, 3 versus 3; reader 2: right AICA, 3 versus 3; left AICA, 3 versus 2) and SCA (reader 1: right SCA, 3 versus 2; left SCA, 2 versus 2; reader 2: right SCA, 4 versus 3 ; left SCA, 3 versus 2) is improved by MBIR compared with FBP.

ture; in-plane resolution was comparable between MBIR and FBP with a "bone" kernel; and image noise was nearly $50 \%$ better by using MBIR than FBP with a "standard" kernel. ${ }^{1}$ Among the 3 different reconstruction kernels, spatial resolution and image noise increase in order from "standard" to "detail" to "bone." Thus, by affecting image noise and spatial resolution, MBIR can improve high- and low-contrast resolution. In addition, MBIR can reduce helical artifacts, including blurring, shading, and windmill artifacts. ${ }^{1}$

The MBIR algorithm that we used in this study was clinically available for cranial CT or CTA examinations only, with a matrix size of $1024 \times 1024$, which also contributed to the increased spatial resolution by MBIR compared with FBP, with a matrix size of $512 \times 512$. Nevertheless, MBIR significantly improved objective image noise compared with FBP.

The mean diameter of the normal basilar artery is $3.17 \mathrm{~mm}$ at the level of the pons. $^{26}$ In phantom experiments, the slope of the profile curve for a vascular model of 3- to 4-mm diameter filled with contrast medium of sufficiently high attenuation became steeper with a smaller pixel size and using a "bone" kernel compared with a "standard" kernel (ie, increased spatial resolution). ${ }^{27,28}$ In this study, this outcome might have led to a significant increase in the attenuation of the BA by MBIR, but we achieved adequate enhancement (eg, >250 HU) in the intracranial arteries even with FBP. With MBIR, the contrast-to-noise ratio increased significantly in the posterior fossa.

On volume-rendered images, all those advantages enhanced image quality and contributed to the significantly improved subjective delineation of all the brain arteries in the posterior fossa except the BA, by MBIR compared with FBP. We observed no significant difference in subjective delineation of the BA between FBP and MBIR, probably because the BA was the largest vessel and sufficiently delineated even with FBP. Actually, the mean visual evaluation score by both readers decreased in order from the BA and VA to the PICA to the SCA to the AICA by using both FBP and MBIR algorithms, and the scores for the BA by FBP were 3 or 4 in all patients except 1 . By MBIR, the mean visual score was 3.0 or higher for all those arteries except the AICA (2.6 \pm 0.7$)$ as assessed by reader 1 , and image quality was acceptable for arteries in the posterior fossa except 1 SCA for reader 1 . On maximum intensity projection compared with volume-rendered imaging, improved vessel enhancement, contrastto-noise ratio and sharpness of vessel contour, and reduced posterior fossa artifacts by MBIR were subjectively more prominent, particu- 
larly in larger arteries such as the BA and VA. On maximum intensity projection, the average improvement of the visual score by MBIR compared with FBP was 0.84 for the BA and VA and 0.46 for the SCA, AICA, and PICA. Compared with FBP, MBIR significantly improved subjective delineation of all brain arteries in the posterior fossa, including the BA. The mean visual score by MBIR was 3.0 or higher for all those arteries except the AICA $(2.8 \pm 0.7$ by both the readers), whereas the score by FBP was 3.0 or higher only for the BA and VA. Thus, MBIR may improve assessment of intracranial aneurysms, especially peripherally located aneurysms of the posterior fossa.

Our study was limited because it was retrospective and included only a small study population. A large-scale prospective study may be warranted to confirm our results. We did we not use DSA images of our patients as a reference for accurately defining the vessels assessed in this study. A final consideration is that we did not directly assess the diagnostic accuracy of intracranial aneurysms, especially in the posterior fossa. However, the close topographic proximity to the osseous structures of the skull base may still make arterial delineation and aneurysm detection challenging even if image quality is substantially improved in the posterior fossa. A further comparison study of diagnostic performance among different reconstruction algorithms and imaging techniques should be performed.

\section{CONCLUSIONS}

In CTA examinations of the head, MBIR is substantially better than FBP in delineating brain arteries in the posterior fossa that are smaller than the BA. Its use may lead to improved noninvasive detection of peripherally located aneurysms of the posterior fossa with CTA and may reasonably reduce radiation and contrast medium dose and contrast injection rate.

\section{ACKNOWLEDGMENTS}

We are grateful to Takuya Hiramoto of GE Healthcare Japan for advice on data interpretation based on the CT reconstruction technologies and his assistance in preparing this manuscript.

Disclosures: Yun Shen-UNRELATED: Employment: GE Healthcare.

\section{REFERENCES}

1. Thibault JB, Sauer KD, Bouman CA, et al. A three-dimensional statistical approach to improved image quality for multislice helical CT. Med Phys 2007;34:4526-44

2. Velthuis BK, Rinkel GJ, Ramos LM, et al. Subarachnoid hemorrhage: aneurysm detection and preoperative evaluation with CT angiography. Radiology 1998;208:423-30

3. Schwartz RB, Tice HM, Hooten SM, et al. Evaluation of cerebral aneurysms with helical CT: correlation with conventional angiography and MR angiography. Radiology 1994;192:717-22

4. Vieco PT, Shuman WP, Alsofrom GF, et al. Detection of circle of Willis aneurysms in patients with acute subarachnoid hemorrhage: a comparison of CT angiography and digital subtraction angiography. AJR Am J Roentgenol 1995;165:425-30

5. Korogi $\mathrm{Y}$, Takahashi M, Katada K, et al. Intracranial aneurysms: detection with three-dimensional CT angiography with volume rendering - comparison with conventional angiographic and surgical findings. Radiology 1999;211:497-506

6. Villablanca JP, Martin N, Jahan R, et al. Volume-rendered helical computerized tomography angiography in the detection and characterization of intracranial aneurysms. J Neurosurg 2000;93:254-64

7. Wiebers DO, Whisnant JP, Huston J 3rd, et al, for the International Study of Unruptured Intracranial Aneurysms Investigators. Unruptured in- tracranial aneurysms: natural history, clinical outcome, and risks of surgical and endovascular treatment. Lancet 2003;362:103-10

8. Ishibashi T, Murayama Y, Urashima M, et al. Unruptured intracranial aneurysms: incidence of rupture and risk factors. Stroke 2009; 40:313-16

9. Hashimoto H, Iida J, Hironaka Y, et al. Use of spiral computerized tomography angiography in patients with subarachnoid hemorrhage in whom subtraction angiography did not reveal cerebral aneurysms. J Neurosurg 2000;92:278-83

10. Kurokawa Y, Yonemasu Y, Kano H, et al. The usefulness of 3D-CT angiography for the diagnosis of spontaneous vertebral artery dissection: report of two cases. Comput Med Imaging Graph 2000;24:115-19

11. Mizushima H, Kobayashi N, Yoshiharu S, et al. Aneurysm of the distal anterior inferior cerebellar artery at the medial branch: a case report and review of the literature. Surg Neurol 1999;52:137-42

12. Carvi y Nievas MN, Haas E, Höllerhage HG, et al. Complementary use of computed tomographic angiography in treatment planning for posterior fossa subarachnoid hemorrhage. Neurosurgery 2002; 50:1283-88, discussion 1288-89

13. Iwanaga $\mathrm{S}$, Shrier DA, Okawara $\mathrm{SH}$, et al. Value of $\mathrm{CT}$ angiography in the evaluation of a peripheral inferior cerebellar artery aneurysm: case report. Clin Imaging 1999;23:77-80

14. Velthuis BK, Van Leeuwen MS, Witkamp TD, et al. Computerized tomography angiography in patients with subarachnoid hemorrhage: from aneurysm detection to treatment without conventional angiography. J Neurosurg 1999;91:761-67

15. Ertl-Wagner BB, Hoffmann RT, Bruning R, et al. Multi-detector row $\mathrm{CT}$ angiography of the brain at various kilovoltage settings. Radiology 2004;231:528-35

16. Ertl-Wagner B, Eftimov L, Blume J, et al. Cranial CT with 64-, 16-, 4and single-slice CT systems-comparison of image quality and posterior fossa artifacts in routine brain imaging with standard protocols. Eur Radiol 2008;18:1720-26

17. Moström U, Ytterbergh C. Artifacts in computed tomography of the posterior fossa: a comparative phantom study. J Comput Assist Tomogr 1986;10:560-66

18. Rozeik C, Kotterer O, Preiss J, et al. Cranial CT artifacts and gantry angulation. J Comput Assist Tomogr 1991;15:381-86

19. Glover GH, Pelc NJ. Nonlinear partial volume artifacts in x-ray computed tomography. Med Phys 1980;7:238-48

20. Joseph PM, Spital RD. A method for correcting bone induced artifacts in computed tomography scanners. J Comput Assist Tomogr 1978;2:100-08

21. Herman GT. Correction for beam hardening in computed tomography. Phys Med Biol 1979;24:81-106

22. Herman GT. Demonstration of beam hardening correction in computed tomography of the head. J Comput Assist Tomogr 1979;3:373-78

23. Elbakri IA, Fessler JA. Statistical image reconstruction for polyenergetic X-ray computed tomography. IEEE Trans Med Imaging 2002; 21:89-99

24. Lin XZ, Miao F, Li JY, et al. High-definition CT gemstone spectral imaging of the brain: initial results of selecting optimal monochromatic image for beam-hardening artifacts and image noise reduction. J Comput Assist Tomogr 2011;35:294-97

25. Hsieh J, Molthen RC, Dawson CA, et al. An iterative approach to the beam hardening correction in cone beam CT. Med Phys 2000;27: 23-29

26. Smoker WR, Price MJ, Keyes WD, et al. High-resolution computed tomography of the basilar artery. 1. Normal size and position $A J N R$ Am J Neuroradiol 1986;7:55-60

27. Suzuki S, Furui S, Kaminaga T. Accuracy of automated CT angiography measurement of vascular diameter in phantoms: effect of size of display field of view, density of contrast medium, and wall thickness. AJR Am J Roentgenol 2005;184:1940-44

28. Suzuki S, Furui S, Kaminaga T, et al. Measurement of vascular diameter in vitro by automated software for CT angiography: effects of inner diameter, density of contrast medium, and convolution kernel. AJR Am J Roentgenol 2004;182:1313-17 